\title{
Harmonic measures for symmetric stable processes
}

\author{
by \\ JANG-MEI Wu (Urbana, IL)
}

\begin{abstract}
Let $D$ be an open set in $\mathbb{R}^{n}(n \geq 2)$ and $\omega(\cdot, D)$ be the harmonic measure on $D^{\mathrm{c}}$ with respect to the symmetric $\alpha$-stable process $(0<\alpha<2)$ killed upon leaving $D$. We study inequalities on volumes or capacities which imply that a set $S$ on $\partial D$ has zero harmonic measure and others which imply that $S$ has positive harmonic measure. In general, it is the relative sizes of the sets $S$ and $D^{\mathrm{c}} \backslash S$ that determine whether $\omega(S, D)$ is zero or positive.
\end{abstract}

We study null sets of harmonic measures for symmetric $\alpha$-stable processes. A symmetric $\alpha$-stable process $X$ on $\mathbb{R}^{n}$ is a Lévy process whose transition density $p(t, x-y)$ relative to Lebesgue measure is uniquely determined by its Fourier transform

$$
\int_{\mathbb{R}^{n}} e^{i x \cdot \xi} p(t, x) d x=e^{-t|\xi|^{\alpha}}
$$

Here $\alpha$ must be in the interval $(0,2]$. When $\alpha=2$, it is a Brownian motion running with a time clock twice as fast as the standard one. From now on, we assume $0<\alpha<2$, when referring to symmetric stable processes. Unlike the Brownian motion, the generator of a symmetric $\alpha$-stable process is nonlocal, as it is the fractional Laplacian $-(-\Delta)^{\alpha / 2}$, an integro-differential operator. A symmetric stable process has discontinuous sample paths and heavy tails, while Brownian motion has continuous sample paths and exponentially decaying tails. Basic properties of symmetric stable processes and their potential-theoretic formulations in terms of Riesz kernels can be found in $[\mathrm{BG}]$ and $[\mathrm{L}]$.

From now on, $D$ is an open set in $\mathbb{R}^{n}(n \geq 2), 0<\alpha<2, X^{D}$ is the symmetric $\alpha$-stable process $X$ killed upon leaving $D$, and $\tau_{D}$ is the first exit time.

An $\alpha$-harmonic function $u$ in $D$ is a locally integrable function in $\mathbb{R}^{n}$ continuous in $D$ satisfying $\int_{|x|>1}|u(x)| \cdot|x|^{-n-\alpha} d x<\infty$ and

$$
u(x)=E^{x} u\left(X_{\tau_{B(x, r)}}\right)
$$

2000 Mathematics Subject Classification: 60J45, 31C99.

The research is supported in part by NSF Grant DMS-0070312. 
for all balls $B(x, r) \subseteq \overline{B(x, r)} \subseteq D ; u$ is said to be regular $\alpha$-harmonic in $D$ if, in addition,

$$
u(x)=E^{x} u\left(X_{\tau_{D}}\right) \quad \forall x \in D,
$$

and $u$ is said to be singular $\alpha$-harmonic if it is $\alpha$-harmonic in $D$ and $u \equiv 0$ in $D^{\mathrm{c}}$. The strong Markov property yields that (0.2) implies (0.1); and if $u$ is $\alpha$-harmonic in $D$ then $u$ is regular $\alpha$-harmonic in $B(x, r)$ whenever $\overline{B(x, r)} \subseteq D$.

For $x \in D$, the $\alpha$-harmonic measure $\omega^{x}(\cdot, D)$ is the measure on $D^{\mathrm{c}}$ given by

$$
\omega^{x}(A, D)=P^{x}\left(X_{\tau_{D}} \in A\right), \quad A \subseteq D^{\mathrm{c}} .
$$

Fix $A \subseteq D^{\mathrm{c}}$, and let $\omega^{x}(A, D)$ be 1 for $x \in A$, and 0 for $x \in D^{\mathrm{c}} \backslash A$; then $\omega^{x}(A, D)$ is regular $\alpha$-harmonic in $D$.

We say $A$ has zero harmonic measure if $\omega^{x}(A, D)=0$ for some $x \in$ $D$, equivalently for all $x \in D$ by the Harnack inequality; we then write $\omega(A, D)=0$.

If $u$ is regular $\alpha$-harmonic in $D$, then

$$
u(x)=\int_{D^{\mathrm{c}}} u(y) d \omega^{x}(y, D) \quad \forall x \in D .
$$

In the case of a ball $B=B(0, r)$, it was shown by M. Riesz that

$$
d \omega^{x}(y, B)=k_{B}(x, y) d y
$$

where

$$
k_{B}(x, y)= \begin{cases}C_{1}(n, \alpha)\left[\frac{r^{2}-|x|^{2}}{|y|^{2}-r^{2}}\right]^{\alpha / 2}|x-y|^{-n}, & |y|>r \\ 0, & |y| \leq r .\end{cases}
$$

Denote by $G$ the Green function of $X$. Then

$$
G(x, y)=\int_{0}^{\infty} p(t, x-y) d t=C_{2}(n, \alpha)|x-y|^{-n+\alpha}
$$

and $G(\cdot, y)$ is $\alpha$-harmonic in $\mathbb{R}^{n} \backslash\{y\}$. The Green function $G_{D}$ of $X^{D}$ is defined by

$$
G_{D}(x, y)=C_{2}(n, \alpha)\left[|x-y|^{-n+\alpha}-\int_{D^{c}}|y-z|^{-n+\alpha} d \omega^{x}(z, D)\right]
$$

note that $G_{D}(x, x)=\infty$ if $x \in D, G_{D}(x, y)=0$ in $(D \times D)^{\mathrm{c}}, G_{D}(x, y)=$ $G_{D}(y, x)$ and $G_{D}(, y)$ is $\alpha$-harmonic in $D \backslash\{y\}$. It is known that for any measurable $f \geq 0$ on $D$,

$$
E^{x}\left[\int_{0}^{\tau_{D}} f\left(X_{s}\right) d s\right]=\int_{D} G_{D}(x, y) f(y) d y \quad \forall x \in D .
$$


For any bounded measurable $\phi \geq 0$ on $D^{\mathrm{c}}$,

$$
E^{x}\left[\phi\left(X_{\tau_{D}}\right): X_{\tau_{D}} \neq X_{\tau_{D^{-}}}\right]=C_{3}(n, \alpha) \int_{D^{\mathrm{c}}} \int_{D} \frac{G_{D}(x, y)}{|y-z|^{n+\alpha}} d y \phi(z) d z,
$$

where $X_{\tau_{D^{-}}}=\lim _{t \uparrow \tau_{D}} X(t)$ exists a.s. ([IW]). Note from $X_{\tau_{D^{-}}} \in \bar{D}$ that

$$
d \omega^{x}(z, D)=C_{3}(n, \alpha)\left(\int_{D} \frac{G_{D}(x, y)}{|y-z|^{n+\alpha}} d y\right) d z \quad \forall x \in D \text { and } z \in \bar{D}^{\mathrm{c}} .
$$

From this it follows that $\omega^{x}(\cdot, D)$ and the Lebesgue measure are mutually absolutely continuous in $\bar{D}^{\mathrm{c}}$.

Consider $S \subseteq \partial D$. If $S$ has positive Lebesgue measure then by (0.4), $\omega^{x}(S, D)>\omega^{x}\left(S, B\left(x, \frac{1}{2} d(x, \partial D)\right)\right)$; and if $S$ has zero $\alpha$-capacity then $\omega^{x}(S, D)=0([\mathrm{~L}])$. Therefore in determining null sets for harmonic measures, it remains to study subsets of the boundary which have positive $\alpha$ capacity and zero volume. (The definition of $\alpha$-capacity will be given in $\S 1$.)

Potential-theoretic problems for $\alpha$-processes for various domains have been studied by Byczkowski, Bogdan, Chen, Kulczycki, Song, and Wu. Bogdan [B1, B2] proved that for a Lipschitz domain $\Omega$, the $\alpha$-harmonic measure of $\partial \Omega$ is zero, the boundary Harnack principle holds and the Martin boundary coincides with the Euclidean boundary. Subsequently, Song and Wu [SW] extended the boundary Harnack principle to all bounded open sets and showed that the Martin boundary and the Euclidean boundary are the same for slit domains. The discontinuity of the processes and the nonlocal definition of harmonicity have imposed many technical complications; on the other hand, the results of Song and Wu suggest that the jumps of the processes make the roughness of $\partial D$ and the disconnectedness of $D$ harmless.

Bogdan's result on harmonic measure says that in a Lipschitz domain $\Omega$, the $\alpha$-processes skip the boundary and jump directly to $\bar{\Omega}^{\mathrm{c}}$ almost surely. It would be interesting to know which other open sets have this property; we have no answer to this question.

In this note, we study inequalities on volumes or capacities which imply that a set $S$ on $\partial D$ has zero harmonic measure, and others which imply a set has positive harmonic measure. In general, it is the relative sizes of the sets $S$ and $D^{\mathrm{c}} \backslash S$ that determine whether $\omega(S, D)$ is positive or zero.

In the following, we use $c$ to denote positive constants depending at most on $n$ and $\alpha$, use $a \gtrsim b$ when $a / b \geq c$ for some $c>0$, and use $a \cong b$ when $a \gtrsim b$ and $b \gtrsim a$. We use $d$ to denote the Euclidean distance, $|S|$ to denote the Lebesgue measure (or volume) of a set $S$ and $\operatorname{dim} S$ to denote the Hausdorff dimension of $S$.

The author thanks R. Song for discussions related to Theorem 1. 
1. Zero harmonic measure. Let $D$ be an open set. We say $D^{\mathrm{c}}$ satisfies the volume density condition (VDC) provided that there exists $c>0$ so that

$$
\left|D^{\mathrm{c}} \cap B(x, 2 d(x, \partial D))\right|>c d(x, \partial D)^{n} \quad \forall x \in D .
$$

We note that in (1.1), balls are centered in the interior of $D$. There is a more commonly used volume density condition $\left(\mathrm{VDC}_{\mathrm{b}}\right)$ which states that there exists $c>0$ so that

$$
\left|D^{\mathrm{c}} \cap B(x, r)\right|>c r^{n} \quad \forall x \in \partial D \text { and } r>0 .
$$

It is easy to check that $\mathrm{VDC}_{\mathrm{b}}$ implies VDC and that $\mathrm{VDC}_{\mathrm{b}}$ implies $|\partial D|=0$. On the other hand, there exist domains $D$ such that $D^{\mathrm{c}}$ satisfies VDC and yet $|\partial D|>0$; for example, let $D$ be a domain having the shape of an infinite branching tree and with branches accumulating at a Cantor set $S$ of positive volume, and having branches chosen so that $D^{\mathrm{c}}$ satisfies VDC.

Our first theorem generalizes Bogdan's result on harmonic measures for Lipschitz domains and possibly has been known to him; however the analytic approach here is more direct.

TheOrem 1. Let $0<\alpha<2$ and $D$ be an open set. If $D^{\mathrm{c}}$ satisfies the volume density condition (VDC) and $\partial D$ has zero volume then $\omega(\partial D, D)=0$.

Theorem 1 follows from the more technical result below.

Theorem $1^{\prime}$. Let $0<\alpha<2, D$ be an open set and $S$ be a measurable subset of $\partial D$ of zero volume. Suppose that there exists $c^{\prime}>0$ so that

$$
\left|D^{\mathrm{c}} \cap B(x, 2 d(x, S))\right|>c^{\prime} d(x, S)^{n} \quad \forall x \in D .
$$

Then $\omega(S, D)=0$.

Proof. Fix $c_{0} \in(0,1 / 2)$, depending only on $n$ and $c^{\prime}$, so that

$$
\left|D^{\mathrm{c}} \cap B(x, 2 d(x, S)) \backslash B\left(x, c_{0} d(x, S)\right)\right|>\frac{c^{\prime}}{2} d(x, S)^{n} \quad \forall x \in D .
$$

For $x \in D$, let $\Delta_{x}=B\left(x, c_{0} \operatorname{dist}(x, S)\right)$. Then by the Markov property and the maximum principle,

$$
\begin{aligned}
\omega^{x}(S, D) & =P^{x}\left\{X_{\tau_{\Delta_{x} \cap D}} \in S\right\}+P\left\{X_{\tau_{\Delta_{x} \cap D}} \in D \backslash \Delta_{x}, X_{\tau_{D}} \in S\right\} \\
& =\omega^{x}\left(S, \Delta_{x} \cap D\right)+\int_{D \backslash \Delta_{x}} \omega^{y}(S, D) d \omega^{x}\left(y, \Delta_{x} \cap D\right) \\
& \leq \omega^{x}\left(S, \Delta_{x}\right)+\sup _{y \in D} \omega^{y}(S, D) \omega^{x}\left(D \backslash \Delta_{x}, \Delta_{x} \cap D\right) \\
& \leq \sup _{y \in D} \omega^{y}(S, D) \omega^{x}\left(D \backslash \Delta_{x}, \Delta_{x}\right) .
\end{aligned}
$$

Note that $\omega^{x}\left(S, \Delta_{x}\right)=0$ because $S$ is contained in $\bar{\Delta}_{x}^{\mathrm{c}}$ and has zero volume. It follows from the integral representation for $\alpha$-harmonic functions in the 
ball $\Delta_{x}((0.3)-(0.5))$ and $(1.3)$ that

$$
\begin{aligned}
\omega^{x}\left(D^{\mathrm{c}} \backslash \Delta_{x}, \Delta_{x}\right) & \geq \omega^{x}\left(D^{\mathrm{c}} \cap B\left(x, 2 d(x, S) \backslash \Delta_{x}\right), \Delta_{x}\right) \\
& \gtrsim\left|D^{\mathrm{c}} \cap B\left(x, 2 d(x, S) \backslash \Delta_{x}\right)\right| d(x, S)^{-n}>c_{0}>0 .
\end{aligned}
$$

Therefore

$$
\sup _{x \in D} \omega^{x}\left(D \backslash \Delta_{x}, \Delta_{x}\right)<1-c_{0}<1,
$$

and it follows from (1.4) that

$$
\sup _{x \in D} \omega^{x}(S, D)<1-c_{0} .
$$

Applying (1.5) and (1.6) to (1.4), we obtain $\omega^{x}(S, D)<\left(1-c_{0}\right)^{2}$ for all $x \in D$. Iterating this process, we obtain $\sup _{D} \omega^{x}(S, D)<\left(1-c_{0}\right)^{k}$ for all $k \geq 1$. Therefore $\omega(S, D)=0$.

In examining the sharpness of (1.2), we arrive at the following example.

EXAmple 1. Let $0<\alpha<2, \lambda>(n+\alpha) /(n-\alpha)$ and $h(t)=\left(\log ^{+}(1 / t)\right)^{-\lambda}$. Then there exist an open set $D$ in $\mathbb{R}^{n}$ and a Cantor set $S \subseteq \partial D$ of dimension $n-\alpha$ such that

$$
\left|D^{\mathrm{c}} \cap B(x, 2 d(x, S))\right| \gtrsim d(x, S)^{n} h(d(x, S)) \quad \forall x \in D ;
$$

however $\omega(S, D)>0$. Moreover $\omega\left(\partial D, \bar{D}^{\mathrm{c}}\right)=0$.

When $n \geq 3$ or when $n=2$ and $0<\alpha \leq 1, \partial D$ can be chosen to be the image of a sphere under a homeomorphism of $\mathbb{R}^{n}$.

A detailed construction will be given in $\S 3$.

We believe that the sharp condition to replace (1.2) in Theorem $1^{\prime}$ lies strictly between (1.2) and (1.7).

Next, we give a sufficient condition for a set $S$ to have zero $\alpha$-harmonic measure by comparing the capacities of $S$ and $D^{\mathrm{c}} \backslash S$.

For $0<\alpha<n$ and $S$ compact in $\mathbb{R}^{n}$, the $\alpha$-capacity of $S, C_{\alpha}(S)$, is

$$
\inf \left\{\mu(S): \int_{S}|x-y|^{-n+\alpha} d \mu(y) \geq 1 \text { on } S\right\} .
$$

There exists a measure $\mu$ ( $\alpha$-capacitory measure) for which the infimum is attained; furthermore $\mu(S)=C_{\alpha}(S)$ and $\int_{S}|x-y|^{-n+\alpha} d \mu(y)=1$ on $S$ except on a set of zero $\alpha$-capacity.

For a Borel set $T$, define

$$
C_{\alpha}(T)=\sup \left\{C_{\alpha}(S): S \text { compact, } S \subseteq T\right\},
$$

and note that

$$
C_{\alpha}(B(0, r)) \cong r^{n-\alpha}
$$


Let $D$ be an open set in $\mathbb{R}^{n}(n \geq 2)$. We say $D^{\mathrm{c}}$ satisfies the $\alpha$-capacity density condition ( $\alpha$-CDC) provided that there exists $\tau>0$ so that

$$
C_{\alpha}\left(D^{\mathrm{c}} \cap \overline{B(x, 2 d(x, \partial D))}\right)>\tau d(x, \partial D)^{n-\alpha} \quad \forall x \in D .
$$

Here again our condition is weaker than the usual one, namely, that there exists $\tau>0$ so that

$$
C_{\alpha}\left(D^{\mathrm{c}} \cap B(x, r)\right)>\tau r^{n-\alpha} \quad \forall x \in \partial D, r>0 .
$$

TheOREm 2. Let $0<\alpha<2, D$ an open set and $S$ a compact subset of $\partial D$. Suppose that $D^{\mathrm{c}}$ satisfies the $\alpha$-capacity density condition (1.8) and $S$ satisfies

$$
\begin{aligned}
C_{\alpha}(S \cap \overline{B(x, 5 d(x, \partial D))}) & \\
<\frac{\tau}{4}\left(2^{-n+\alpha}-3^{-n+\alpha}\right) d(x, \partial D)^{n-\alpha} & \forall x \in D .
\end{aligned}
$$

Then $\omega(S, D)=0$.

The theorem says that if the $\alpha$-capacity of $D^{\mathrm{c}}$ is large and the $\alpha$-capacity of $S$ is small in comparison at all scales then $\omega(S, D)=0$.

Proof. Let $T=D^{\mathrm{c}} \backslash S$, and for a given $x \in D$, let $c B=B(x, c d(x, \partial D))$. Note from (1.8) and (1.9) that $C_{\alpha}(T \cap \overline{2 B})>\frac{3}{4} \tau d(x, \partial D)^{n-\alpha}$. Choose a compact subset $\widetilde{T}$ of $T \cap \overline{2 B}$ so that $C_{\alpha}(\widetilde{T})>(\tau / 2) d(x, \partial D)^{n-\alpha}$ and let $\mu$ be its $\alpha$-capacitory measure.

Let $\Omega=(D \cap 5 B) \cup((5 B \backslash \overline{2 B}) \backslash S)$ and note that $\Omega^{c}=(T \cap \overline{2 B}) \cup(S \cap$ $5 B) \cup(5 B)^{\mathrm{c}}$. It follows from the maximum principle that

$$
\omega^{x}(T, D) \geq \omega^{x}(T \cap \overline{2 B}, \Omega) .
$$

Since $\omega(T \cap \overline{2 B}, \Omega)+\omega(S \cap 5 B, 5 B \backslash S)$ and $\omega(\widetilde{T}, 5 B \backslash \widetilde{T})$ are $\alpha$-harmonic in $\Omega$ and

$$
\omega(T \cap \overline{2 B}, \Omega)+\omega(S \cap 5 B, 5 B \backslash S) \geq \omega(\widetilde{T}, 5 B \backslash \widetilde{T})
$$

in $\Omega^{\mathrm{c}}$, the inequality (1.10) holds at $x$ also.

Then, by the conditions imposed on $\widetilde{T}$,

$$
\begin{aligned}
& \omega^{x}(\widetilde{T}, 5 B \backslash \widetilde{T}) \\
& \quad \geq \int_{\widetilde{T}}|x-y|^{-n+\alpha} d \mu(y)-\int_{(5 B)^{\mathrm{c}} \widetilde{T}}|z-y|^{-n+\alpha} d \mu(y) d \omega^{x}(z, 5 B) \\
& \quad \geq \int_{\widetilde{T}}|x-y|^{-n+\alpha} d \mu(y)-(3 \operatorname{dist}(x, \partial D))^{-n+\alpha} \mu(\widetilde{T}) \\
& \quad \geq\left(2^{-n+\alpha}-3^{-n+\alpha}\right) d(x, \partial D)^{-n+\alpha} \mu(\widetilde{T}) \geq\left(2^{-n+\alpha}-3^{-n+\alpha}\right) \tau / 2 .
\end{aligned}
$$


Let $\nu$ be the $\alpha$-capacity measure for $S \cap \overline{5 B}$. Then

$$
\begin{aligned}
\omega^{x}(S \cap \overline{5 B}, 5 B \backslash S) & \leq \int_{S \cap 5 B}|x-y|^{-n+\alpha} d \mu(y) \\
& \leq \mu(S \cap \overline{5 B}) d(x, \partial D)^{-n+\alpha} .
\end{aligned}
$$

From (1.9)-(1.12) and the subadditivity of capacity it follows that

$$
\omega^{x}(T, D) \geq \frac{\tau}{4}\left(2^{-n+\alpha}-3^{-n+\alpha}\right) \quad \forall x \in D,
$$

or

$$
\omega^{x}(S, D)<c(n, \alpha, \tau)<1 \quad \forall x \in D .
$$

Therefore $\omega(S, D)=0$ by Lemma 1 below.

Let $\mathcal{H}_{D}$ be the class of functions continuous in $\mathbb{R}^{n}$ and $\alpha$-harmonic in $D, C\left(D^{\mathrm{c}}\right)$ be the class of functions $f$ continuous in $D^{\mathrm{c}}$ satisfying $\int_{D^{\mathrm{c}} \cap\{|x|>1\}}|f(x)| \cdot|x|^{-n-\alpha} d x<\infty$, and $H(D)$ be the class of functions on $\mathbb{R}^{n}, \alpha$-harmonic in $D$. Following Landkof ([L], Chap. IV, 15 and 21), there exists a unique positive linear operator $A$ from $C\left(D^{\mathrm{c}}\right)$ into $H(D)$ so that for $f \in \mathcal{H}_{D}, A\left(f \mid D^{\mathrm{c}}\right)=f$ in $\mathbb{R}^{n}$; moreover

$$
A f(x)=\int_{D^{\mathrm{c}}} f(y) d \omega^{x}(y, D) \text { on } D,
$$

and $A f(x)$ is the unique (up to a set of $\alpha$-capacity zero) $\alpha$-harmonic function in $D$ which has limit $f(z)$ at each regular boundary point $z$. The set of irregular boundary points has zero $\alpha$-capacity, hence zero $\alpha$-harmonic measure; and $A f$ is called the solution of the Dirichlet problem for the given $f$.

Given $f \in C\left(D^{\mathrm{c}}\right)$, let $\mathcal{P}_{f}$ (Perron family) be the class of functions $u, \alpha$ subharmonic in $D$, with $u \leq f$ in $\bar{D}^{\mathrm{c}}$ and boundary values $u^{*} \leq f$ on $\partial D$. (An $\alpha$-subharmonic function in $D$ is a function locally integrable in $\mathbb{R}^{n}$, upper semicontinuous in $D$ satisfying $\int_{|x|>1}|u(x)| \cdot|x|^{-n-\alpha} d x<\infty$ and $u(x) \leq E^{x} u\left(X_{\tau_{B(x, r)}}\right)$ for all balls $B(x, r) \subseteq \overline{B(x, r)} \subseteq D$; and $u^{*}(z) \equiv$ $\lim \sup u(x)$ as $x \rightarrow z$ in $D$ for $z \in \partial D$.) Define

$$
L f(x)=\sup \left\{u(x): u \in \mathcal{P}_{f}\right\} \quad \text { in } \mathbb{R}^{n} .
$$

Following the argument for Brownian motion $(\alpha=2)$ as in $[\mathrm{F}]$ or $[\mathrm{H}]$, we can prove that $L f$ is $\alpha$-harmonic in $D$, and $L$ is a positive linear operator from $C\left(D^{\mathrm{c}}\right)$ into $H(D)$ with $A\left(f \mid D^{\mathrm{c}}\right)=f$ for $f \in \mathcal{H}_{D}$. By the uniqueness, $L=A$.

The lemma below is known for the Brownian motion $(\alpha=2)$. When $0<\alpha<2$, it is less apparent and is false if the assumption $S \subseteq \partial D$ is removed; note for example that if $D=B(0,1)$ and $S=\{2 \leq|x| \leq 3\}$, then $\sup _{x \in D} \omega^{x}(S, D)<1$ and $\omega^{0}(S, D)>0$. 
Lemma 1. Let $0<\alpha<2, D$ be an open set and $S$ be a compact subset of $\partial D$. Suppose that $\sup _{x \in D} \omega^{x}(S, D)<1$. Then $\omega(S, D)=0$.

Proof. Let $f_{k}$ be a decreasing sequence of continuous functions on $D^{\mathrm{c}}$ with $0 \leq f_{k} \leq 1, \operatorname{supp} f_{k} \downarrow S$ and $f_{k} \downarrow \chi_{S}$. Then

$$
A f_{k}(x)=\int_{D^{\mathrm{c}}} f_{k}(y) d \omega^{x}(y, D) \downarrow \omega^{x}(S, D) \quad \text { on } D .
$$

Let $a=\sup _{x \in D} \omega^{x}(S, D)<1$. We claim that for each $k$,

$$
\omega^{x}(S, D) \leq a A f_{k}(x) \text { on } D .
$$

This shows that $\omega^{x}(S, D) \leq a \omega^{x}(S, D)$ on $D$, which implies that $\omega(S, D)=0$.

To prove (1.13), we fix $k$ and let

$$
v(x)=\omega^{x}(S, D)-a A f_{k}(x) \quad \text { in } \mathbb{R}^{n} .
$$

Note that $v \leq 1$ and that $v^{*}(z) \leq 0$ at every regular boundary point $z$. Take $\varepsilon>0$ and let $F_{\varepsilon}$ be the set of boundary points $z$ where $v^{*}(z) \geq \varepsilon$. The set $F_{\varepsilon}$ is closed and consists of irregular points only, hence $\omega\left(F_{\varepsilon}, D\right)=0$. Choose a decreasing sequence of continuous functions $g_{j}$ on $D^{\mathrm{c}}$ with $0 \leq g_{j} \leq 1$, $\operatorname{supp} g_{j} \downarrow F_{\varepsilon}$ and $g_{j} \downarrow \chi_{F_{\varepsilon}}$. Then $v \in \mathcal{P}_{\varepsilon+g_{j}}$ for each $j$. Therefore

$$
v(x) \leq L\left(\varepsilon+g_{j}\right)(x)=\varepsilon+L g_{j}(x)=\varepsilon+A g_{j}(x)
$$

for each $j$. Since $A g_{j}(x) \downarrow \omega^{x}\left(F_{\varepsilon}, D\right)=0$ in $D$, we have $v(x) \leq \varepsilon$ in $D$ for any $\varepsilon>0$. This proves the claim and thus the lemma.

\section{Positive harmonic measure}

Theorem 3. Let $0<\alpha<2, D$ be an open set and $S$ be a compact subset of $\partial D$ having positive $\alpha$-capacity. Suppose that $C_{\alpha}\left(\left\{x \in D^{\mathrm{c}}: 0<\right.\right.$ $d(x, S) \leq a\}) \rightarrow 0$ as $a \rightarrow 0$. Then $\omega(S, D)>0$.

Proof. We assume as we may that $S \subseteq B(0,1 / 2)$. Let $T=D^{\mathrm{c}} \backslash S$, and let $T_{a}=\left\{x \in D^{\mathrm{c}}: 0<d(x, S) \leq a\right\}$ and $T_{a, b}=\left\{x \in D^{\mathrm{c}}: b \leq d(x, S) \leq a\right\}$ for $0<b<a<1 / 10$. Then

$$
C_{\alpha}\left(T_{a}\right)=\sup _{b>0} C_{\alpha}\left(T_{a, b}\right)
$$

Choose and fix $a>0$ so that

$$
C_{\alpha}\left(T_{a}\right)<2^{-n+\alpha-1}\left(1-2^{-n+\alpha}\right) C_{\alpha}(S),
$$

and let $b \in(0, a)$. Denote by $\mu$ and $\nu$ the $\alpha$-capacitory measures of $S$ and $T_{a, b}$ respectively. Let

$$
U(x)=\int_{S}|x-y|^{-n+\alpha} d \mu(y), \quad V(x)=\int_{T_{a, b}}|x-y|^{-n+\alpha} d \nu(y) .
$$


Recall that $U$ and $V$ are positive $\alpha$-harmonic in $\mathbb{R}^{n} \backslash S$ and $\mathbb{R}^{n} \backslash T_{a, b}$ respectively, with $U \equiv 1$ on $S$ and $V \equiv 1$ on $T_{a, b}$ except possibly on some sets of zero $\alpha$-capacity, and $\mu(S)=C_{\alpha}(S)$ and $\nu\left(T_{a, b}\right)=C_{\alpha}\left(T_{a, b}\right)$. Hence

$$
\nu\left(T_{a, b}\right)<2^{-n+\alpha-1}\left(1-2^{-n+\alpha}\right) \mu(S) .
$$

Note that for $|x| \geq 3$,

$$
\begin{aligned}
V(x) & \leq(|x|-1)^{-n+\alpha} \nu\left(T_{a, b}\right) \\
& <(|x|-1)^{-n+\alpha} 2^{-n+\alpha-1}\left(1-2^{-n+\alpha}\right) \mu(S) \\
& <2^{-n+\alpha-1}\left(1-2^{-n+\alpha}\right)\left(\frac{|x|-1}{|x|+1}\right)^{-n+\alpha} U(x) \\
& \leq 2^{-1}\left(1-2^{-n+\alpha}\right) U(x) .
\end{aligned}
$$

Define $u(x)=\omega^{x}(S, B(0,10)), v(x)=\omega^{x}\left(T_{a, b}, B(0,10)\right)$ and let $\widetilde{u}$ be the function in $\mathbb{R}^{n} \alpha$-harmonic in $B(0,10)$ with $\widetilde{u} \equiv U$ on $B(0,10)^{\text {c }}$, i.e.

$$
\widetilde{u}(x)=\int_{B(0,10)^{\mathrm{c}}} U(y) d \omega^{x}(y, B(0,10)) \quad \text { in } B(0,10) .
$$

Note that

$$
u(x)>U(x)-\widetilde{u}(x) \quad \text { on } B(0,10) .
$$

Since $U(y) \leq 9^{-n+\alpha} \mu(S)$ for $|y| \geq 10$, we have $\widetilde{u} \leq 9^{-n+\alpha} \mu(S)$. Therefore for $|x|<10$,

$$
\begin{aligned}
u(x) & >U(x)-9^{-n+\alpha} \mu(S) \\
& =\left(1-2^{-n+\alpha}\right) U(x)+2^{-n+\alpha} U(x)-9^{-n+\alpha} \mu(S) .
\end{aligned}
$$

Note that for $|x|=3, U(x)>4^{-n+\alpha} \mu(S)$, and

$$
u(x)>\left(1-2^{-n+\alpha}\right) U(x)>2 V(x)>2 v(x)
$$

from (2.1). Therefore for $|x|=3$,

$$
\begin{aligned}
\omega^{x}\left(S \cup T_{b}, B(0,10) \backslash\left(S \cup T_{a}\right)\right) & \geq \omega^{x}\left(S, B(0,10) \backslash\left(S \cup T_{a, b}\right)\right) \\
& \geq u(x)-v(x)>\frac{1}{2} u(x) \\
& >\frac{1}{2}\left(1-2^{-n+\alpha}\right) U(x)>c(n, \alpha) \mu(S)
\end{aligned}
$$

for some $c(n, \alpha)>0$. Letting $b \rightarrow 0^{+}$, we obtain

$$
\omega^{x}\left(S, B(0,10) \backslash\left(S \cup T_{a}\right)\right) \geq c(n, \alpha) \mu(S) \text { for }|x|=3 .
$$

Hence by Lemma 1 ,

$$
\sup \left\{\omega^{x}\left(S, B(0,10) \backslash\left(S \cup T_{a}\right)\right): x \in B(0,10) \backslash\left(S \cup T_{a}\right)\right\}=1 .
$$

If $T_{a}=T \cap B(0,10)$, then $B(0,10) \backslash\left(S \cup T_{a}\right)=D \cap B(0,10)$, thus

$$
\omega^{x}(S, D) \geq \omega^{x}(S, B(0,10) \cap D)>0 \quad \text { in } D \cap B(0,10) .
$$


If $T \cap B(0,10) \backslash T_{a} \neq \emptyset$, let

$$
A=\sup \left\{\omega^{x}\left(S, B(0,10) \backslash\left(S \cup T_{a}\right)\right): x \in B(0,10) \cap T \backslash T_{a}\right\} .
$$

Because $\operatorname{dist}\left(S, B(0,10) \cap T \backslash T_{a}\right) \geq a>0$, we have $0<A<1$. It follows from (2.2) that there exists $x_{0} \in B(0,10) \backslash\left(S \cup T_{a}\right)$ so that $\omega^{x_{0}}(S, B(0,10) \backslash$ $\left.\left(S \cup T_{a}\right)\right)>A$. Since $D^{\mathrm{c}}$ is the union of two disjoint sets $S \cup T_{a}$ and $T \backslash T_{a}$, it follows from the Markov property and the choice of $A$ and $x_{0}$ that

$$
\begin{aligned}
& \omega^{x_{0}}(S, B(0,10) \cap D)=\omega^{x_{0}}\left(S, B(0,10) \backslash\left(S \cup T_{a}\right)\right) \\
& \quad-\int_{B(0,10) \cap T \backslash T_{a}} \omega^{x}\left(S, B(0,10) \backslash\left(S \cup T_{a}\right)\right) d \omega^{x_{0}}\left(x, B(0,10) \backslash\left(S \cup T_{a}\right)\right)>0 .
\end{aligned}
$$

This implies that $\omega^{x_{0}}(S, D)>0$ and proves the theorem.

The proof of Theorem 3 shows in fact the following.

Theorem $3^{\prime}$. There exists $c(n, \alpha)>0$ so that for any open set $D$ and compact set $S \subseteq \partial D$, if $C_{\alpha}(S)>0$ and

$$
C_{\alpha}\left(\left\{x \in D^{\mathrm{c}}: 0<d(x, S) \leq a\right\}\right)<c(n, \alpha) C_{\alpha}(S)
$$

for some $a>0$, then $\omega(S, D)>0$.

3. Examples. Our theorems suggest that it is the relative sizes of $S$ and $D^{\mathrm{c}} \backslash S$ that determine the vanishing of $\omega(S, D)$. Examples 1 and 2 reinforce this observation, and use all three theorems in the reasoning.

First we give the details for Example 1.

Since $\lambda>(n+\alpha) /(n-\alpha)$, we have $(\lambda+1)(n-\alpha) / n>2$. Choose $\varepsilon, \delta, a_{k}, x_{k}$ so that $0<\delta<\varepsilon<(\lambda+1)(n-\alpha) / n-2$ and

$$
a_{k}^{n-\alpha}=2^{-n k} k^{1+\delta} \quad \text { and } \quad x_{k}^{n-\alpha}=k^{-2-\varepsilon} .
$$

Calculations show that

$$
k x_{k}^{n}=k^{1-(2+\varepsilon) n /(n-\alpha)}>k^{-\lambda}
$$

and for large $k$, say $k \geq k_{0}$,

$$
2 a_{k+1}+3 x_{k} a_{k}<a_{k} .
$$

Let $S$ be the Cantor set in the unit cube constructed as follows. Let $Q_{0}^{1}=[0,1]^{n}$. Assume that the cubes $Q_{k}^{j}\left(1 \leq j \leq 2^{n k}\right)$ of side length $a_{k}$ have been constructed. Inside each $Q_{k}^{j}$, choose $2^{n}$ closed subcubes of side length $a_{k+1}$ at the vertices of $Q_{k}^{j}$. Call this collection of subcubes $Q_{k+1}^{i}(1 \leq$ $\left.i \leq 2^{n(k+1)}\right)$. Let

$$
S=\bigcap_{k} \bigcup_{j} Q_{k}^{j}
$$


Let $B_{k}^{j}$ be the closed ball concentric with $Q_{k}^{j}$ of radius $x_{k} a_{k}$ and let

$$
D=\mathbb{R}^{n} \backslash\left(S \cup \bigcup^{\prime} B_{k}^{j}\right)
$$

where $\bigcup^{\prime} B_{k}^{j}$ denotes the union $\bigcup_{k \geq k_{0}} \bigcup_{j} B_{k}^{j}$. Note that $\partial D=S \cup \bigcup^{\prime} \partial B_{k}^{j}$.

The volume condition (1.7) follows easily from the estimate

$$
\begin{aligned}
\left|D^{\mathrm{c}} \cap Q_{k}^{j}\right| & =\sum_{m=k}^{\infty} 2^{n(m-k)}\left|B_{m}^{1}\right| \cong \sum_{m=k}^{\infty} 2^{-n k} 2^{n m} x_{m}^{n} a_{m}^{n} \\
& \cong k x_{k}^{n} a_{k}^{n} \geq k^{-\lambda} a_{k}^{n}
\end{aligned}
$$

It is clear that $\operatorname{dim} S=n-\alpha$.

To see $C_{\alpha}(S)>0$, we let $\mu$ be the measure on $S$ so that $\mu\left(S \cap Q_{k}^{j}\right)=2^{-k n}$ and observe that $\mu(S \cap B(x, t)) \leq C(n) t^{n-\alpha}\left(\log \frac{2 \sqrt{n}}{t}\right)^{-1-\delta}$ for any $x \in S$ and $0<t \leq \sqrt{n}$. Therefore

$$
\int_{S}|x-y|^{-n+\alpha} d \mu(y) \lesssim \int_{0}^{\sqrt{n}} \frac{\mu(B(x, t))}{t^{n-\alpha+1}} d t \leq c(n, \alpha, \delta)<\infty
$$

for all $x \in S$; this proves that $C_{\alpha}(S)>0$.

We now prove that

$$
C_{\alpha}\left(S \cap Q_{k}^{j}\right) \cong 2^{-k n} k^{\delta}=a_{k}^{n-\alpha} k^{-1},
$$

a fact needed in later discussion. Consider the restriction of $\mu$ (from the last paragraph) onto $Q_{k}^{j}$; then for any $x \in S \cap Q_{k}^{j}$,

$$
\int_{S \cap Q_{k}^{j}}|x-y|^{-n+\alpha} d \mu(y) \lesssim \int_{0}^{\sqrt{n} a_{k}} \frac{\mu(B(x, t))}{t^{n-\alpha+1}} d t \cong k^{-\delta} .
$$

For each $x \in S \cap Q_{k}^{j}$, a mutually disjoint sequence $\left\{Q_{m}^{i(m, x)}: m \geq k+1\right\}$ of cubes in $Q_{k}^{j}$ can be found so that $\operatorname{dist}\left(x, Q_{m}^{i(m, x)}\right) \cong a_{m}$; therefore

$$
\begin{aligned}
\int_{S \cap Q_{k}^{j}}|x-y|^{-n+\alpha} d \mu(y) & \gtrsim \sum_{m=k+1}^{\infty} \operatorname{dist}\left(x, Q_{m}^{i(m, x)}\right)^{-n+\alpha} \mu\left(Q_{m}^{i(m, x)}\right) \\
& \cong \sum_{m=k+1}^{\infty} a_{m}^{-n+\alpha} 2^{-m n} \cong k^{-\delta}
\end{aligned}
$$

Since $\mu\left(S \cap Q_{k}^{j}\right)=2^{-k n}$, this proves $(3.1)$. 
Define $W_{k}=\bigcup_{m \geq k} \bigcup_{j} B_{m}^{j}$ for $k \geq k_{0}$. Then by the subadditivity of capacities,

$$
\begin{aligned}
C_{\alpha}\left(W_{k}\right) & \leq \sum_{m=k}^{\infty} \sum_{j} C_{\alpha}\left(B_{m}^{j}\right) \lesssim \sum_{m=k}^{\infty} 2^{n m} x_{m}^{n-\alpha} a_{m}^{n-\alpha} \\
& \leq \sum_{m=k}^{\infty} m^{-1-\varepsilon+\delta} \cong k^{-\varepsilon+\delta} .
\end{aligned}
$$

In fact $C_{\alpha}\left(W_{k}\right) \cong k^{-\varepsilon+\delta}$; this is not needed in our argument and the detail is not given.

Since $C_{\alpha}(S)>0$ and $C_{\alpha}\left(W_{k}\right) \rightarrow 0$ as $k \rightarrow \infty$, it follows from Theorem 3 that $\omega(S, D)>0$.

Since the exterior of the open set $\bar{D}^{\mathrm{c}}$ satisfies the VDC and $\partial D$ has zero volume, it follows from Theorem 1 that $\omega\left(\partial D, \bar{D}^{\mathrm{c}}\right)=0$.

When $n \geq 3$, or when $n=2$ and $0<\alpha \leq 1$, a line segment has zero $\alpha$-capacity. Fix a closed ball $B_{0}$ centered at $(1 / 2, \ldots, 1 / 2)$ disjoint from $S \cup \bigcup^{\prime} B_{k}^{j}$. First connect $B_{0}$ to each $B_{k_{0}}^{i}$ by a thin wire $L_{k_{0}}^{i}$ in the unit cube and then connect each $B_{k}^{j}\left(k \geq k_{0}\right)$ to every $B_{k+1}^{i}$ contained in $Q_{k}^{j}$ by a thin wire $L_{k+1}^{i}$ in $Q_{k}^{j}$ so that every wire meets exactly two balls, the entire collection of wires are mutually disjoint and $S \cup B_{0} \cup \cup^{\prime}\left(B_{k}^{j} \cup L_{k}^{j}\right)$ is the image of a closed ball under a global homeomorphism. Let

$$
D=\mathbb{R}^{n} \backslash\left(S \cup B_{0} \cup \bigcup^{\prime}\left(B_{k}^{j} \cup L_{k}^{j}\right)\right) \text {. }
$$

When wires are chosen thin enough, all assertions in Example 1 are still valid.

ExAMPLE 2. If we retain $S$ from Example 1 and replace each $B_{k}^{j}$ by a closed set in $Q_{k}^{j} \backslash \bigcup_{i} Q_{k+1}^{i}$ having $\alpha$-capacity $\cong a_{k}^{n-\alpha}$, then, in contrast with Example 1, $\omega(S, D)=0$. This follows from Theorem 2, the statement (3.1) and the fact that for the new set $D, D^{\mathrm{c}}$ satisfies $\alpha$-CDC.

\section{References}

[BG] R. M. Blumenthal and R. K. Getoor, Markov Processes and Potential Theory, Academic Press, 1968.

[B1] K. Bogdan, The boundary Harnack principle for the fractional Laplacian, Studia Math. 123 (1997), 43-80.

[B2] - Representation of $\alpha$-harmonic functions in Lipschitz domains, Hiroshima Math. J. 29 (1999), 227-243.

[F] W. H. J. Fuchs, Topics in the Theory of Functions of One Complex Variable, Van Nostrand, Princeton, 1967.

[H] L. L. Helms, Introduction to Potential Theory, Wiley-Interscience, New York, 1969. 
[IW] N. Ikeda and S. Watanabe, On some relations between the harmonic measure and the Lévy measure for a certain class of Markov processes, J. Math. Kyoto Univ. 2 (1962), 79-95.

[L] N. S. Landkof, Foundations of Modern Potential Theory, Springer, Berlin, 1972.

[SW] R. Song and J. M. Wu, Boundary Harnack principle for symmetric stable processes, J. Funct. Anal. 169 (1999), 403-427.

Department of Mathematics

University of Illinois

1409 West Green Street

Urbana, IL 61801, U.S.A.

E-mail: wu@math.uiuc.edu

Received December 28, 2000

Revised version July 30, 2001 